IV.

\title{
Bemerkungen
}

\section{zu einer Aufgabe der Wahrscheinlichkeitsrechnung.}

[Journal für reine und angewandte Mathematik, Bd.50, S. 268-271 (1855)].

In einem der früheren Hefte des "Philosophical Magazine" für 1854 hat G. Boole eine von A. Cayley gegebene Auflösung einer Wahrscheinlichkeitsaufgabe angegriffen. Wiewohl nicht zu zweifeln ist, daß der in diesem Angriff enthaltene Irrtum auch von anderen schon erkannt sei, so kommen doch die folgenden Bemerkungen denen, welche sich für diese schöne Theorie interessieren, vielleicht nicht unerwünscht.

Die Aufgabe lautet: Gegeben ist die Wahrscheinlichkeit $\alpha$, daß eine Ursache $A$ (welche ein gewisses Ereignis hervorbringen kann) zur Wirkung kommt, und die Wahrscheinlichkeit $p$, daß, wenn $A$ wirkt, das Ereignis eintritt; ebenso die Wahrscheinlichkeit $\beta$, daß eine Ursache $B$ zur Wirkung gelangt, und die Wahrscheinlichkeit $q$, daß, wenn $B$ wirkt, das Ereignis eintritt: gesucht wird die Wahrscheinlichkeit $u$ des Ereignisses, unter der Annahme, daß dasselbe von keiner anderen Ursache als von $A$ und $B$ hervorgebracht werden kann.

Cayley löset die Aufgabe auf folgende Weise: Es sei $\lambda$ die Wahrscheinlichkeit, daß, wenn $A$ wirkt, das Ereignis auch durch $A$ hervorgebracht wird; $\mu$ die Wahrscheinlichkeit, daß, wenn $B$ wirkt, das Ereignis auch durch $B$ hervorgebracht wird; dann ist

$$
p=\lambda+(1-\lambda) \mu \beta, \quad q=\mu+(1-\mu) \lambda \alpha .
$$

Hierans werden $\lambda, \mu$ bestimmt; und die gesuchte Wahrscheinlichkeit ist

$$
u=\lambda \alpha+\mu \beta-\lambda \mu \alpha \beta .
$$

Nachdem nun Boole diese Auflösung bei mehreren Spezialisierungen als richtig bewährt fand, sucht er nachzuweisen, daß sie 
in dem Falle $p=1, q=0$ zu einem falschen Resultat führe. Er sagt: Es ist einleuchtend, daß die Wahrscheinlichkeit des Ereignisses in diesem Falle $=\alpha$ sein muß. Denn wenn die Ursache $A$ das Ereignis stets hervorbringt, die Ursache $B$ niemals, und das Eintreten des Ereignisses keiner anderen Ursache zugeschrieben werden kann, so muß die Wahrscheinlichkeit des „Ereignisses gleich der des Eintretens der Ursache $A$ sein". Da sich gegen diesen Satz natürlich nichts einwenden läßt, und nun die Auflösung, wie sie Cayley darstellt, in diesem Falle entweder $u=1$ oder $u=\alpha(1-\beta)$ gibt, so schließt Boole, daß die ganze Auflösung fehlerhaft sein müsse, und gibt die Endformel seiner eigenen Auflösung, mit Hinzufügung besonderer Beschränkungen, aus denen sich allerdings für diesen Fall das gewünschte Resultat $u=\alpha$ ableiten läßt.

Man sieht indessen durchaus nicht, wo Cayley einen Fehler gemacht hätte; und in der Tat ist seine Auflösung auch (bis auf gewisse Beschränkungen, durch welche sie erst eindeutig gemacht werden muß) streng richtig, selbst in dem eben angeführten Falle; denn man findet leicht, daß $\alpha(1-\beta)$ mit $\alpha$ übereinstimmt, indem $\alpha$ nichts anderes als Null sein kann. Wäre nämlich die Möglichkeit des Eintretens der Ursache $A$ offen gelassen, d. h. wäre $\alpha$ nicht Null so könnte auch unmöglich die Wahrscheinlichkeit $q$ des Ereignisses (unter der Annahme des Eintretens der Ursache $B$ ) gänzlich verschwinden, mag $p$ noch so klein, nur nicht Null sein (in diesem Falle war aber $p=1$ angenommen). Die gestellte Aufgabe ist daher widersinnig, wenn $q=0, \alpha$ und $p$ dagegen beide von Null verschieden angenommen werden. Dies ergibt sich auch durch einen Blick auf die Gleichungen 'von Cayley. Wenn man nämlich beachtet, daß $\mu,(1-\mu), \lambda, \alpha$, der Natur ihrer Bedeutung nach, nicht negativ sein können, so folgt aus der einen Gleichung $q=0$, sowohl $\mu=0$, als auch $\lambda \alpha=0$, und die andere Gleichung geht in $p=\lambda$ über. Ist nun $p$ von Null verschieden (es ist nicht nötig, daß $p$ gerade $=1$ sei), so muß auch $\alpha=0$ sein; und die gesuchte Wahrscheinlichkeit $u$ muß stets $=0$ sein, mag $q$ oder $p$, oder mögen beide $=0$ sein; wie man es nicht anders erwarten darf.

Wenn nun aber dieser Vorwurf auch die obige Auflösung nicht trifft, so ist sie doch wenigstens noch unvollständig zu nennen, da die Bedingungen nicht angegeben sind, unter welchen die Aufgabe wirklich einen reellen Sinn hat, und da ferner zu entscheiden übrig 
bleibt, welchen der beiden Werte von $u$, die den obigen Gleichungen genügen, man zu wählen habe. Dies soll hier geschehen.

Man verfährt mit der meisten Symmetrie, wenn man $\mu$ aus den Gleichungen für $q$ und $u$, und ebenso $\lambda$ aus den Gleichungen für $p$ und $u$ eliminiert. Dies gibt

$$
u-\beta q=(1-\beta) \lambda \alpha, \quad u-\alpha p=(1-\alpha) \mu \beta,
$$

und wenn man diese Werte von $\lambda \alpha, \mu \beta$ in die Gleichung für $u$ substituiert, so erhält man eine quadratische Gleichung, durch deren Auflösung sich

$$
u=\frac{1}{2}(1-\alpha \beta+\alpha p+\beta q-\varrho)
$$

ergibt, worin $\varrho$ die noch zweideutige Quadratwurzel aus

$$
\begin{aligned}
\varrho \varrho= & (1-\alpha \beta+\alpha p+\beta q)^{2}-4(1-\beta) \alpha p, \\
& -4(1-\alpha) \beta q-4 \alpha p \cdot \beta q
\end{aligned}
$$

ist. Damit aber die Aufgabe lösbar sei, ist nötig: zuerst, daß $\varrho$ reell, und weiter, daß $u$ (als eine Wahrscheinlichkeit) ein positiver echter Bruch sei. Aber auch dies ist noch nicht genügend; und darin liegt eigentlich das Hauptinteresse der ganzen Aufgabe. Sie würde immer noch ohne Sinn bleiben, wenn die Hilfswahrscheinlichkeiten $\lambda, \mu$ nicht ebenfalls zwischen den Grenzen 0 und 1 enthalten wären, und es ist klar, daß mit diesen letzten Bedingungen auch zugleich die ersten erfüllt werden müssen. Es kommt daher nur darauf an, die Bedingungen aufzustellen, welche ausdrücken, daß $\lambda, \mu$ nicht außerhalb der genannten Grenzen liegen. Dies ist leicht, da man die Werte $\lambda, \mu$ aus den Gleichungen (1) erhält, wenn man in ihnen für $u$ den in (2) gefundenen Ausdruck substituiert. Bei dieser Untersuchung kommt man auf die folgenden Gleichungen:

$$
\begin{aligned}
\varrho \varrho & =(1-2 \alpha+\alpha \beta+\alpha p-\beta q)^{2}+4 \alpha(1-\alpha)(1-\beta)(1-p) \\
& =(1-2 \beta+\alpha \beta-\alpha p+\beta q)^{2}+4 \beta(1-\beta)(1-\alpha)(1-q) \\
& =(1-\alpha \beta+\alpha p-\beta q)^{2}-4 \alpha(1-\beta)(p-\beta q) \\
& =(1-\alpha \beta-\alpha p+\beta q)^{2}-4 \beta(1-\alpha)(q-\alpha p) .
\end{aligned}
$$

Aus den beiden ersten Formen für $\varrho \varrho$ geht hervor, daß es keiner besonderen Bedingung für die Realität von $\varrho$ bedarf. Setzt man aber die Formen in Verbindung mit den Forderungen für $\lambda$, $\mu$, so ergibt sich, daß in dem Ausdrucke (2) für $u$ stets die positive Quadratwurzel für $\varrho$ genommen werden muß. Vergleicht man endlich die beiden letzten Formen für $\varrho \varrho$ mit den Forderungen für $\lambda$ und $\mu$, so 
erhält man, als die einzigen notwendig erforderlichen, aber auch vollständig genügenden Bedingungen, daß die beiden Differenzen

$$
p-\beta q \text { and } q-\alpha p
$$

nicht negativ sein dürfen.

Wenn man also, um ein Beispiel zu der Aufgabe zu geben, für $\alpha, \beta, p, q$ vier beliebige Zahlen innerhalb der Grenzen 0 und 1 angenommen hat, so muß man erst untersuchen, ob sie den beiden Bedingungen in (3) Genüge leisten. Beiläufig bemerkt, kann man bei einer solchen willkürlichen Wahl ebensooft ein widersinniges Beispiel wie ein passendes treffen; denn der Wert des vierfachen Integrals $\iiint \int d \alpha d \beta d p d q$ ist $=1$, wenn man die. Integrationen über alle Werte der Veränderlichen zwischen 0 und 1 ausdehnt; dagegen $=\frac{1}{2}$, wenn man diejenigen Werte ausschließt, welche den Bedingungen (3) nicht Genüge leisten. Man kann sich hiervon auch leicht durch geometrische Betrachtungen überzeugen.

In dem von Boole untersuchten Falle $q=0$ reduzieren sich die Bedingungen (3) auf $\alpha p=0$; dann wird $\varrho=1-\alpha \beta$, und folglich $u=0$, ganz in Übereinstimmung mit den obigen Resultaten. Auch der Fall $\alpha=0$ ist von Interesse. Dann ist $\varrho=1-\beta q$, und folglich $u=\beta q$ offenbar das richtige Resultat. Hierbei ist natürlich $u$ von $q$ unabhängig, und dennoch bleibt die Bedingung $p-\beta q \geqq 0$ in voller Kraft, und obgleich zur Bestimmung von $u$ auf den Wert von $p$ gar kein Gewicht fällt, so wäre es doch widersinnig, die Wahrscheinlichkeit $p$ des Ereignisses unter der Annahme, daß die Ursache $A$ zur Wirkung gelangt, kleiner als die Wahrscheinlichkeit $\beta q$ anzunehmen, wenn auch diese Annahme durch die Bestimmung $\alpha=0$ faktisch verboten ist. Und mit dieser Bemerkung, die, wie ich glaube, dazu geeignet ist, auf die Eigentümlichkeit dieser Art von Aufgaben ein frappantes Licht zu werfen, will ich meine Betrachtungen abbrechen.

Göttingen, 22. Juli 1854. 